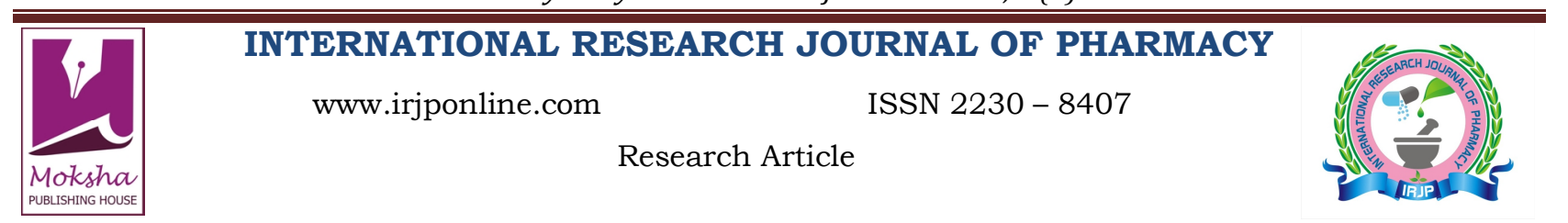

\title{
STUDY ON THE EFFICACY OF IODINE BASED CONTRAST MEDIA FOR INTERPRETATION OF ROOT CANAL ANATOMY
}

Shetty Aditya, Hegde Mithra, Tahiliani Divya*, Joshi Aum, Devadiga Darshana

Department of Conservative Dentistry \& Endodontics, A B Shetty Memorial Institute of Dental Sciences, Nitte University

Mangalore, Karnataka, India

Email: divya.tahiliani@gmail.com

Article Received on: 20/01/13 Revised on: 01/02/13 Approved for publication: 13/03/13

DOI: $10.7897 / 2230-8407.04344$

IRJP is an official publication of Moksha Publishing House. Website: www.mokshaph.com

(C) All rights reserved.

\section{ABSTRACT}

Root canal anatomy in the human dentition is very complex. The radiographic identification of these root canal features may assist in the assessment and treatment of potential endodontic failures. The technique described here introduces a radiopaque contrast medium into the root canal system for better visualization. A new contrast media includes Iopamidol (Non Ionic Monomer) which till date is not used in dentistry. This chemical is used in Angiographic indication, Urography, Computed Tomography and Venography. 24 extracted teeth were collected. Access cavity was prepared on all of them. The teeth were divided into 2 groups and different contrast media (also with $\mathrm{NaOCl}+$ EDTA) were introduced into the canal. The individual teeth were radiographed. The radiographs were scored. Significant difference was seen between the 4 subgroups. The newer contrast media which is non -ionic iodine based monomer with low viscosity and low osmolality when used in combination with a cleansing and a chelating agent, served as a very effective contrast media in endodontics.

Key Words: Root canal anatomy, Radiopaque contrast medium, Iopamidol, NaOCl, EDTA, Endodontics.

\section{INTRODUCTION}

The human tooth has a complex pulp space anatomy. The presence of patent furcal, lateral and accessory canals are the portals of entry and exit between the root canal space and the periodontal ligament ${ }^{1,2}$. Radiographic identification of these portals may aid in assessment of anatomy of pulpal system. The radiographic interpretation of pulp space anatomy suffers from superimposition of surrounding bone tissue ${ }^{3}$.

All tooth roots have a main canal: these are the canals which are instrumented and obturated during endodontic treatment. Although the apical foramina of the roots are the principal pathways between the pulp and the periodontal ligament, patent lateral and accessory canals may exist. These canals may form a communication between the pulp and PDL, permitting either irritants from the necrotic pulp to irritate and inflame the lateral PDL, or in case of a periodontal lesion, toxic substances to enter the pulp to induce pathological changes. (W.B.Saunders 1989, R.C Burns 1991) $)^{4}$ The premolars especially mandibular, show a more complex root canal anatomy than any other tooth. ${ }^{4,5}$

The radiographic identification of these root canal features may assist in the assessment and treatment of potential endodontic failures. Studies have shown that radiographs often fail to provide the basic information that is required such as the number of canals within the tooth or the presence of lateral canals. $^{4}$

A method which enhances the appearance of the canals may therefore be considered of value in endodontic therapy. The technique of introducing a radiopaque contrast medium into the root canal system of extracted permanent teeth was used by Barker et.al (1973) to investigate the anatomy of pulp space from radiographs. Numerous radiopaque contrast media have been developed and used to various degree of success. $^{4}$

Many methods have been used to investigate root canal anatomy. In vitro each has its own advantages and disadvantages with variable degrees of accuracy and procedural complexity. ${ }^{5}$

In order to overcome these adverse effects, a newer contrast media is used in the present study. The contrast media includes Iopamidol (MILANO ITALY Bracco s.p.a) (Non Ionic Monomer), which has not been used in clinical dentistry so far. This chemical is used in Angiographic indication, Urography, Computed Tomography and Venography.

\section{MATERIALS AND METHODS}

Twenty four extracted intact human mandibular premolars in each group (12 first premolars \& 12 Second premolars) were stored in $10 \%$ sodium hypochlorite. Each tooth was radiographed in the mesio-distal planes using DDDIOR (DDDIOR-Direct Digital Dental Intra Oral Radiography) system.

Standardized endodontic access cavities were prepared using a high -speed hand piece with a diamond point bur and water coolant.

On location of the pulp chambers with a patency file of ISO no $6 \mathrm{~K}$ File, the canals were progressively enlarged to 8,10 and 15, after which gross pulpal debris were removed using barbed broaches. The prepared individual teeth were then correctly oriented and mounted using wax in a modified plaster phantom.

The prepared individual teeth were divided into two main groups.

GROUP 1 Contrast media was introduced into the canal, and GROUP 2 Contrast media+ sodium hypochlorite + EDTA (COMDENT Corp. Mumbai) was introduced into the canal.

Contrast media was placed in a hot water bath and then immediately introduced intra pulpally into each of the prepared tooth.

Another contrast media Iopromide (Manus Aktteva Biopharma LLP) was also used. A syringe and an ISO no 15 $\mathrm{K}$-file was used to ensure that the media reached the apex. The individually mounted teeth were radiographed (DDDIOR). The images were presented to three viewers and scoring was done. 

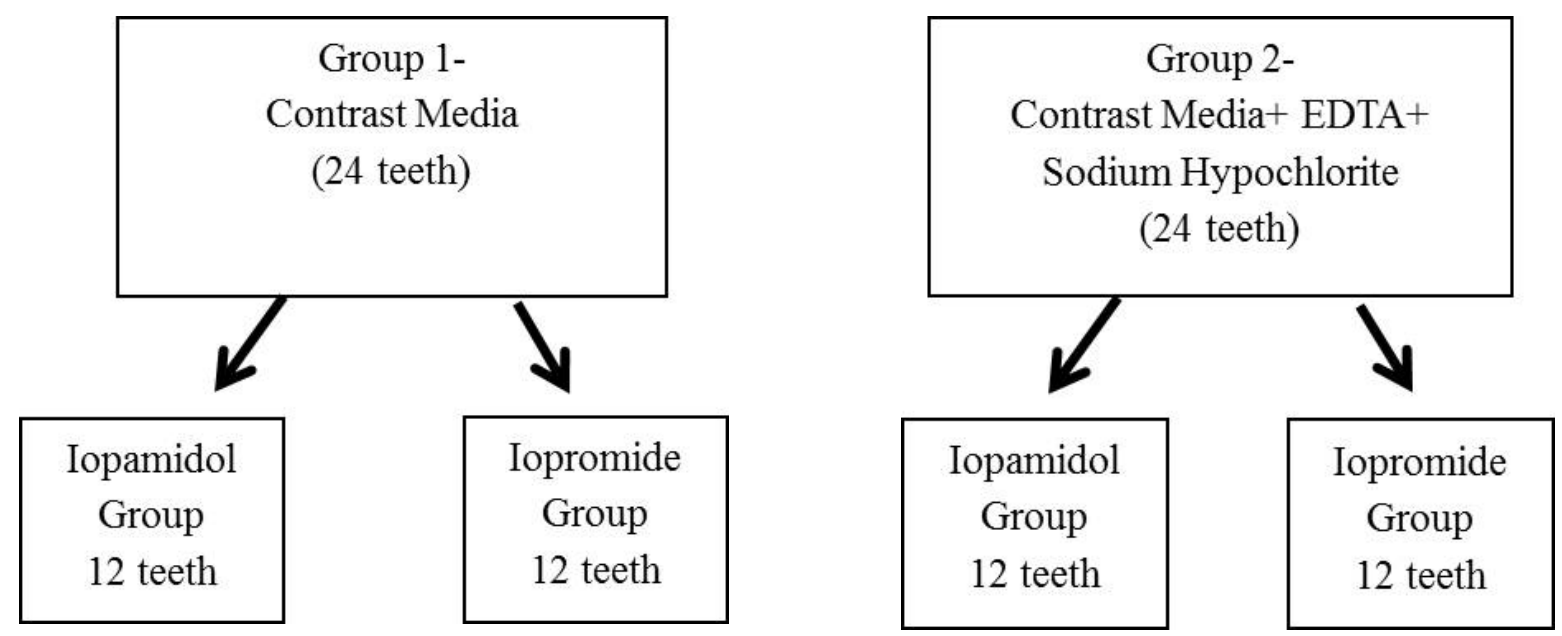

Table 1: Values recorded as per assessment by three viewers in Group 1

\begin{tabular}{|c|c|c|c|c|c|c|}
\hline \multicolumn{7}{|c|}{ Group 1 - Contrast Media } \\
\hline & \multicolumn{3}{|c|}{ Iopamidol } & \multicolumn{3}{c|}{ Iopromide } \\
\hline Teeth No & Viewer 1 & Viewer 2 & Viewer 3 & Viewer 1 & Viewer 2 & Viewer 3 \\
\hline 1 & 1 & 1 & 1 & 2 & 2 & 2 \\
\hline 2 & 0 & 0 & 0 & 1 & 1 & 1 \\
\hline 3 & 2 & 2 & 1 & 1 & 1 & 0 \\
\hline 4 & 1 & 1 & 1 & 1 & 1 & 1 \\
\hline 5 & 1 & 1 & 2 & 2 & 2 & 2 \\
\hline 6 & 0 & 0 & 0 & 1 & 1 & 1 \\
\hline 7 & 1 & 1 & 1 & 0 & 0 & 0 \\
\hline 8 & 0 & 0 & 0 & 1 & 1 & 1 \\
\hline 9 & 1 & 1 & 1 & 2 & 2 & 2 \\
\hline 10 & 2 & 2 & 0 & 1 & 2 & 1 \\
\hline 11 & 0 & 1 & 2 & 1 & 0 & 1 \\
\hline 12 & 1 & 1 & 0 & 1 & 1 & 1 \\
\hline
\end{tabular}

Table 2: Values recorded as per assessment by threee viewers in Group 2

\begin{tabular}{|c|c|c|c|c|c|c|}
\hline \multicolumn{7}{|c|}{ Group 2 - Contrast Media + NaOCI + EDTA } \\
\hline & \multicolumn{3}{|c|}{ Iopamidol } & \multicolumn{3}{c|}{ Iopromide } \\
\hline Teeth No & Viewer 1 & Viewer 2 & Viewer 3 & Viewer 1 & Viewer 2 & Viewer 3 \\
\hline 1 & 3 & 3 & 3 & 3 & 3 & 3 \\
\hline 2 & 2 & 2 & 2 & 3 & 3 & 3 \\
\hline 3 & 2 & 2 & 2 & 3 & 3 & 3 \\
\hline 4 & 3 & 3 & 3 & 3 & 3 & 3 \\
\hline 5 & 3 & 3 & 3 & 2 & 2 & 1 \\
\hline 6 & 3 & 3 & 3 & 3 & 3 & 3 \\
\hline 7 & 2 & 2 & 2 & 1 & 1 & 0 \\
\hline 8 & 1 & 1 & 1 & 3 & 3 & 3 \\
\hline 9 & 3 & 2 & 2 & 3 & 3 & 2 \\
\hline 10 & 2 & 3 & 3 & 2 & 3 & 3 \\
\hline 11 & 2 & 2 & 2 & 1 & 2 & 3 \\
\hline 12 & 3 & 2 & 2 & 3 & 3 & 2 \\
\hline
\end{tabular}

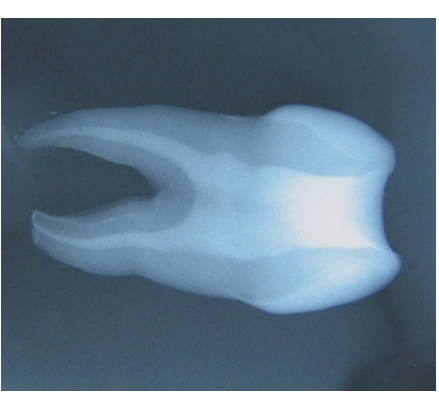

Figure 1: Radiograph clearly showing the root canal morphology in Group 2 Sample, Subgroup Iopamidol

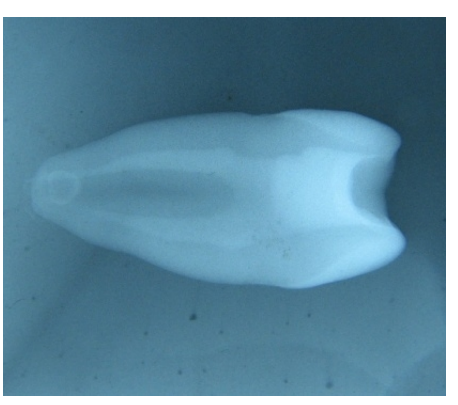

Figure 2: Radiograph clearly showing the root canal morphology in Group 2 Sample, Subgroup Iopromide

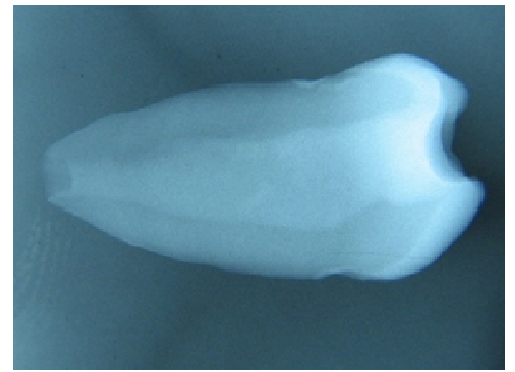

Figure 3: Radiograph clearly showing the root canal morphology in Group 2 Sample, Subgroup Iopromide 


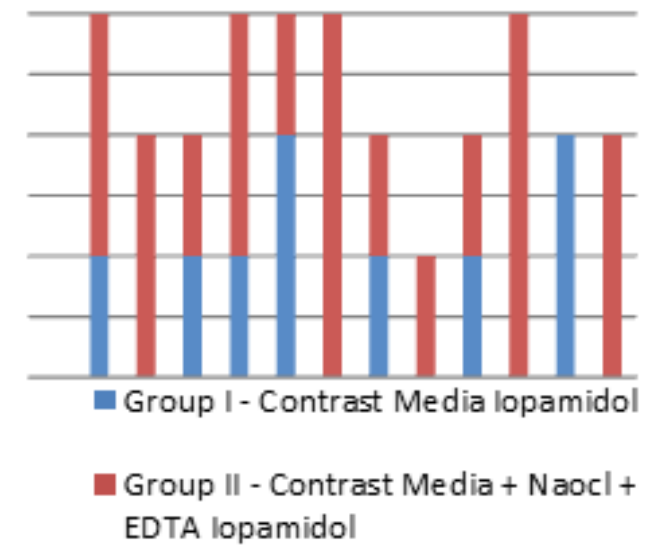

Figure 4: Group 2 has better properties than Group 1
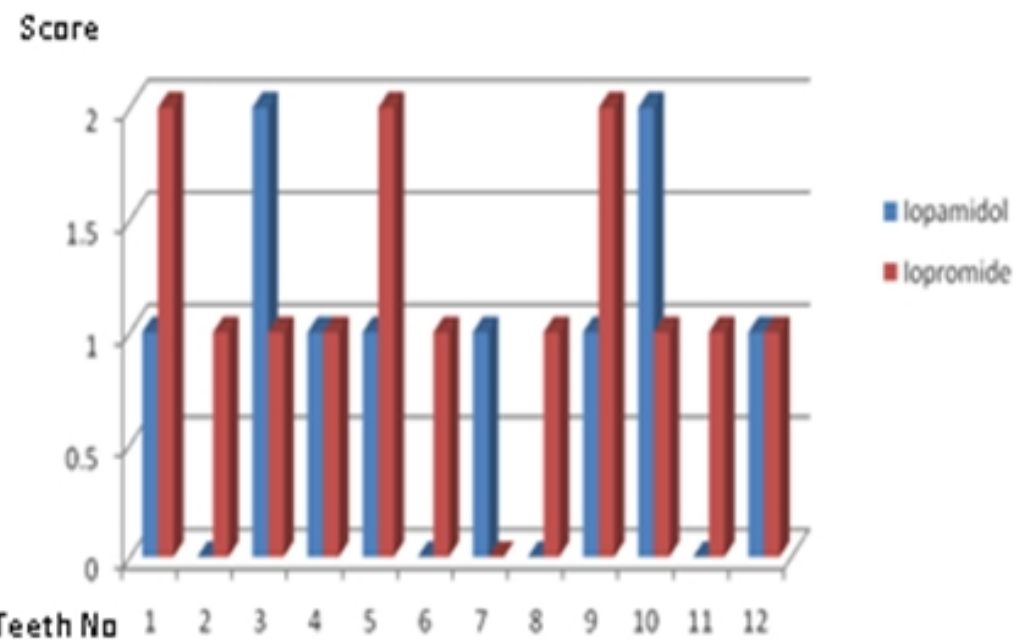

Figure 5: Iopamidol and Iopromide have almost the same properties

Scoring criteria

$0=$ No Penetration of dye in to the root canal of the tooth

$1=$ Penetration of dye into the root canal up to the middle part of the root canal.

$2=$ Penetration of dye into the root canal up to the apical part of the root canal.

$3=$ Penetration of dye into the root canal up to the apical part of the root canal along with penetration into the lateral and the accessory canals of the same.

Inter group comparison was done using statistical analysis.

\section{RESULTS}

The observation has been summarized in Table 1 and 2 .

Intergroup comparison

Group 1- Contrast media

Group 2- Contrast media + sodium hypochlorite + EDTA

Contrast Media used

1) Iopamidol

2) Iopromide

KRUSKAL- WALLIS test was done between them for Group 1 and for Group 2

For Group 1

P- Value for Iopamidol $=0.817$

P- Value for Iopromide $=0.935$
For Group 2

P- Value for Iopamidol $=0.919$

P- Value for Iopromide $=0.489$

The result showed that for $\mathrm{P}>0.05$, no statistically significant difference between the contrast media (Iopamidol and Iopromide) were seen.

MANN-WHITNEY test was done between Group 1 and Group 2 for Iopamidol \& Iopromide separate.

For both the contrast media the $\mathrm{P}$ value was less than 0.001

The result showed a significant difference between the Group 1 (Contrast Media) and Group 2(Contrast Media $+\mathrm{NaOCl}+$ EDTA)

\section{DISCUSSION}

Good quality radiographs are the single most important aid in detecting extra roots and canals in endodontic diagnosis . The ability to accurately markdown the possibility of an extra canal would be clinically useful to rule out, the riskof iatrogenic damage associated with trying to locate the perceived additional canal. Also on gaining access to the root canals, the contrast medium could be used to verify the canal morphology pre-operatively.

The use of radiopaque contrast medium in endodontic radiography may be a valuable aid in the diagnosis and 
evaluation of root canal system. This system would complement rather than replace plain radiography.

In the present in vitro study a simple technique was used to introduce two radiopaque contrast media into the root canal system of extracted teeth under negative pressure.

In previous studies by J.M Bedford et.al, only the dye was introduced into the root canal after pulp extirpation, but the presence of pulp remnants normally hindered the proper flow of the dye into the canal.

Here the sodium hypochlorite portion of the composition will digest the pulp and eliminate bacteria and the related irritants within the root canal system thus enabling the iodine portion of the composition to flow into the vacated space. EDTA, a chelating agent was used to remove the inorganic part in the canal thereby increasing the flow.

The viscosity of the contrast media also played a small role in the reduced flow. Therefore, the formulation was changed to increase the efficiency of the dye. Hence the preparationused was a solution of contrast media $+5 \%$ sodium hypochlorite + $17 \%$ EDTA.

The solution was placed in a thermostatically controlled water bath (to reduce the viscosity). The results showed that a combination of Iopromide $+5 \%$ Sodium Hypochlorite + EDTA showed better flow and radio opacity as compared with Iopamidol. This could be due to;less viscosity and hence better flow characteristic of Iopromid. Better visualization was achieved due to presence of higher iodine content.

\section{CONCLUSION}

The contrast media, non- ionic iodine based monomer with low viscosity and low osmolality when used in combination with a cleansing and a chelating agent, served as a very effective contrast media in endodontics. From this invitro study it can be inferred that the non- ionic iodine based contrast media Iopromide and Iopamidol, both were effective when used as an adjunct in routine endodontic radiography. Iopromide when used along with a cleansing agent and EDTA had better characteristics of flow and contrast in assessment of root canal anatomy. However additional studies are needed to be carried out to further investigate in this front. This reflects the need to understand the complex root canal anatomy better.

\section{ACKNOWLEDGEMENT}

The authors deny any conflict of interest related to this study.

\section{REFERENCES}

1. Torabinejad M. Endodontic/periodontic interrelationships. In: Walton RE, Torabinejad M,eds. Principles and practice of endodontics. 4th ed. Philadelphia; WB Saunders 2009:94-107

2. Frank J. Vertucci, James E. Haddix. Tooth Morphology and Access
Cavity Preparation. In:Cohen S, Burns RC, eds Pathways of the Pulp. 10th ed. St. Louis; Mosby Year Book, 2010:136-222

3. Fan B, Gao Y, Fan W, Gutmann J. Identification of a C-shaped canal system in mandibular second molars - part ii: the effect of bone image superimposition and intraradicular contrast medium on radiograph interpretation. J Endod. 2008;34(2):160-165 http://dx.doi.org/10.1016 /i.joen.2007.10.010 PMid: 18215673

4. Naoum, H. J, Love, R. M, Chandler, N. P and Herbison, P. Effect of Xray beam angulation and intraradicular contrast medium on radiographic interpretation of lower first molar root canal anatomy. International Endodontic Journal. 2003;36:12-19. http://dx.doi.org/10.1046/j.01432885.2003.00604.x PMid:12656509

A. C. Shearer, F Wasti and N. H. F. Wilson. The use of a radiopaque contrast medium in endodontic radiography. International Endodontic Journal. 1996; 29[2]:95 - 98. http://dx.doi.org/10.1111/j.13652591.1996.tb01168.x PMid:9206431

5. W. Scarfe, C. Fana Jr., A. Farman. Radiographic detection of accessory/lateral canals: Use of RadioVisioGraphy and hypaque. Journal of Endodontics. 1995; 21[4]:185-190. http://dx.doi.org/10.1016/S00992399(06)80563-7

6. N. D. Pugh. Haemodynamic and rheological effects of contrast media: the role of viscosity and osmolality. Journal European Radiology. 1996, April; 6[2]

7. Hession RW. Endodontic morphology: An alternative method of study. Oral Surg Oral Med Oral Pathol. 1997 Sep; 44[3]:456-462. http:/ /dx.doi.org/10.1016/0030-4220(77)90416-9

8. Tayfun Alcam, Guliz Gorgul and Human Omurulu. Evaluation of diagnostic radiopaque contrast materials used with calcium. Journal of Endodontics. 1999; 16[8]:365-368. http://dx.doi.org/10.1016/S00992399(06)81907-2

9. R. De Moor, F. Calberson. Root Canal Treatment in a Mandibular Second Premolar with Three Root Canals. Journal of Endodontics. 2005; 31[4]:310-313. http://dx.doi.org/10.1097/01.don.0000140578.36109.c0 PMid: 15793392

10. B. Fan, Y. Gao, W. Fan, J. Gutmann. Identification of a C-shaped Canal System in Mandibular Second Molars-Part II: The Effect of Bone Image Superimposition and Intraradicular Contrast Medium on Radiograph Interpretation. Journal of Endodontics. 2008; 34[2]:160-165. http://dx.doi.org/10.1016/j.joen.2007.10.010 PMid:18215673

11. Katayama H, Yamaguchi K, Kozuka T, Takashima T, Seez P, Matsuura $\mathrm{K}$. Adverse reactions to ionic and nonionic contrast media. A report from the Japanese Committee on the Safety of Contrast Media. Dentomaxillofacial Radiology. 1998; 27:199-202

12. Metzger, M. Solomonov, E. Mass. Calcium hydroxide retention in wide root canals with flaring apices. Dental Traumatology. 2001; 17[2]:86-92. http://dx.doi.org/10.1034/j.1600-9657.2001.017002086.x PMid:11475951

13. S. A. Beyer-Enke and E. Zeitler. Late adverse reactions to non-ionic contrast media: a cohort analytic study. European Radiology. 1993; 3:237-241. http://dx.doi.org/10.1007/BF00425901

14. Saeed Rahimi,Shahriar Shahi, Hamid Reza Yavari, Hakimeh Manafi, Nemat Eskandarzadeh, Root Canal Configuration of Mandibular First and Second Premolars in an Iranian Population. Journal of Dental Research, Dental Clinics, Dental Prospects, 2007; 1[2]

15. Stephen Cohen, Richard C. Burns, Pathways of Pulp, 8th Edition. St. Louis: Mosby; 2002. p 261

\section{Cite this article as:}

Shetty Aditya, Hegde Mithra, Tahiliani Divya, Joshi Aum, Devadiga Darshana. Study on the efficacy of iodine based contrast media for interpretation of root canal anatomy. Int. Res. J. Pharm. 2013; 4(3):207-210 